\title{
FREE RESOLUTIONS FOR CERTAIN CLASSES OF GROUPS
}

\author{
by SUBRATA MAJUMDAR
}

(Received 12th July 1981)

\section{Introduction}

In a previous paper [1] we constructed a free resolution for a class of groups which include Fuchsian groups with compact orbit spaces [2, 3], infinite polyhedral groups, plane crystallographic groups $p^{2}, p^{3}, p^{4}$ and $p^{6}$ and Dyck's groups [4], and used this resolution for computation of the integral homology and cohomology of these groups. Lyndon [5] determined the cohomology of groups with a single defining relation. The plane crystallographic groups $p^{1}$ and $p g$ and Artin's braid group $B_{3}$ are among these groups. In this paper we have constructed free resolutions for certain classes of groupsresolutions which are particularly suitable for direct computation of the homology and the cohomology of these groups for any coefficient module. These classes of groups include the plane crystallographic groups $\mathrm{pm}, \mathrm{cm}$ and $\mathrm{pgg}$. We have computed the integral homology and cohomology from each of the free resolutions obtained.

Let $G$ be a group given by $G=F / R$, where $F$ is a free group generated freely by $x_{1}, x_{2}, \ldots, x_{m}$ and $R$ is the normal closure of $r_{1}, r_{2}, \ldots, r_{n} \in F$. Lyndon $[5,6]$ showed that a free $\mathbb{Z} G$-resolution of $\mathbb{Z}$ can be started as follows:

$$
\ldots \rightarrow \rightarrow Y_{1} \stackrel{d_{1}}{\rightarrow} Y_{0} \stackrel{d_{0}}{\rightarrow} \mathbb{Z} G \stackrel{\varepsilon}{\rightarrow} \mathbb{Z} \rightarrow 0
$$

where $Y_{0}$ is a right $\mathbb{Z} G$-module free on $\alpha_{1}, \alpha_{2}, \ldots, \alpha_{m}$,

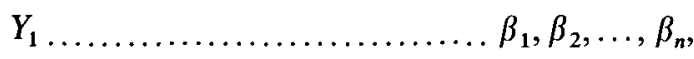

and the homomorphisms are given by

$$
\begin{aligned}
& \varepsilon(g)=1 \in \mathbb{Z}, \text { for each } g \in G, \text { extended by linearity, } \\
& d_{0}\left(\alpha_{i}\right)=h_{i}-1, \quad i=1,2, \ldots, m, \\
& d_{1}\left(\beta_{j}\right)=\sum_{i=1}^{m} \alpha_{i} \pi\left(\frac{\partial r_{j}}{\partial x_{i}}\right), \quad j=1,2, \ldots, n,
\end{aligned}
$$

each extended by $\mathbb{Z} G$-linearity, where $\pi: \mathbb{Z} F \rightarrow \mathbb{Z} G$ is the homomorphism induced by the 
canonical homomorphism $F \rightarrow \frac{F}{R}=G$, each $h_{i}=\pi\left(x_{i}\right)$, and each $\frac{\partial r_{j}}{\partial x_{i}}$ is defined by

$$
r_{j}-1=\sum_{i=1}^{m}\left(x_{i}-1\right) \frac{\partial r_{j}}{\partial x_{i}},(\text { see }[7])
$$

Each of the free resolutions we have constructed is an extension of (1).

We shall use two results, viz. Propositions 1 and 2, of [1] in this paper and they are all that we need for our proofs. For the sake of completeness, we state these two results together as Proposition 1 below.

Let $H$ be a subgroup of a group $G$ and $\bar{H}$ a set of generators of $H$. For any subset $S$ of $\mathbb{Z} G$, let Ann ${ }_{R} S$ denote the right annhilator of $S$.

Define

$$
\begin{aligned}
s(H) & =\sum_{h \in H} h, \quad \text { if } H \text { is finite } \\
& =0, \quad \text { otherwise. }
\end{aligned}
$$

Proposition 1. ([1])

$$
\begin{aligned}
& \text { If } H \text { is finite, } \operatorname{Ann}_{R}\{\mathrm{~s}(H)\}=\sum_{h \in h}(h-1) \cdot \mathbb{Z} G . \\
& \bigcap_{h \in \bar{H}} \operatorname{Ann}_{R}\{h-1\}=s(H) \cdot \mathbb{Z} G .
\end{aligned}
$$

In this paper we have used the symbol $s_{j}$ to denote the element $1+h_{j}+\ldots+h_{j}^{m_{j}-1}$ of $\mathbb{Z} G$, where $h_{j}$ is an element of order $m_{j}$ in a group $G$.

\section{2.}

Let $G$ be a group given by

generators: $\quad h_{1}, h_{2}, \ldots, h_{k}, h_{k+1}$;

relations: $\quad h_{1}^{m_{1}}=\ldots=h_{k}^{m_{k}}=e$,

$$
h_{1} \dot{h_{k+1}}=h_{k+1} h_{1}, \ldots, h_{l} h_{k+1}=h_{k+1} h_{l}(l \leqq k)
$$

If $k=l=2$, and $m_{1}=m_{2}=2, G \cong p m([4]$, p. 136). 


\section{Definition 1.}

$$
\cdots \stackrel{\sigma}{\rightarrow} Y \stackrel{\mathfrak{\tau}}{\rightarrow} Y \stackrel{\sigma}{\rightarrow} Y \stackrel{\tau}{\rightarrow} Y \stackrel{\sigma}{\rightarrow} Y \stackrel{d_{1}}{\rightarrow} Y_{0} \stackrel{d_{0}}{\rightarrow} \rightarrow G \stackrel{\varepsilon}{\rightarrow} \mathbb{Z} \rightarrow 0,
$$

is a sequence of right $\mathbb{Z} G$-modules and $\mathbb{Z} G$-homomorphisms defined as follows:

$$
\begin{aligned}
& Y_{0} \text { is a right } G \text {-module free on } \alpha_{1}, \alpha_{2}, \ldots, \alpha_{k+1} \\
& Y \ldots \ldots \ldots \ldots \ldots \ldots \ldots \ldots \ldots \ldots, \beta_{1}, \beta_{2}, \ldots, \beta_{k+l}
\end{aligned}
$$

and the homomorphisms are given by

$\varepsilon(g)=1 \in \mathbb{Z}$, for each $g \in G$, and extended by linearity;

$d_{0}\left(\alpha_{i}\right)=h_{i}-1, \quad i=1,2, \ldots, k, \quad k+1$;

$d_{1}\left(\beta_{j}\right)=\alpha_{j} s_{j}, \quad j=1,2, \ldots, k$

$d_{1}\left(\beta_{k+j^{\prime}}\right)=\alpha_{j^{\prime}}\left(h_{k+1}-1\right)-\alpha_{k+1}\left(h_{j^{\prime}}-1\right), \quad j^{\prime}=1,2, \ldots, l ;$

$\sigma\left(\beta_{j}\right)=\beta_{j}\left(h_{j}-1\right), \quad j=1,2, \ldots, k$

$\sigma\left(\beta_{k+j^{\prime}}\right)=\beta_{k+j^{\prime}} s_{j^{\prime}}-\beta_{j^{\prime}}\left(h_{k+1}-1\right), \quad j^{\prime}=1,2, \ldots, l ;$

$\tau\left(\beta_{j}\right)=\beta_{j} s_{j}, \quad j=1,2, \ldots, k$

$\tau\left(\beta_{k+j^{\prime}}\right)=\beta_{k+j^{\prime}}\left(h_{j^{\prime}}-1\right)+\beta_{j^{\prime}}\left(h_{k+1}-1\right), \quad j^{\prime}=1,2, \ldots, l ;$

and each extended by $\mathbb{Z} G$-linearity.

Theorem 1. The sequence (2) is a free $\mathbb{Z} G$-resolution of $\mathbb{Z}$.

Proof. Since (2) is an extension of (1), it will be sufficient to verify the exactness of (2) only at the fifth, the sixth and the seventh terms from the right.

The verification of the fact that the image of each homomorphism is contained in the kernel of the following homomorphism is straightforward.

We only have to show that (i) $\operatorname{Ker} d_{1} \subseteq \operatorname{Im} \sigma$, (ii) $\operatorname{Ker} \sigma \subseteq \operatorname{Im} \tau$, and (iii) $\operatorname{Ker} \tau \subseteq \operatorname{Im} \sigma$.

First, let $\gamma=\sum_{s=1}^{k+l} \beta_{s} \gamma_{s} \in \operatorname{Ker} d_{1}$. Then, since $Y_{0}$ is free on $\alpha_{1}, \alpha_{2}, \ldots, \alpha_{k+1}$, it follows that

$$
\begin{aligned}
& s_{1} \gamma_{1}+\left(h_{k+1}-1\right) \gamma_{k+1}=0 \\
& s_{l} \gamma_{l}+\left(h_{k+1}-1\right) \gamma_{k+1}=0 \\
& s_{l+1} \gamma_{l+1}=0 \\
& \vdots \quad \vdots \\
& s_{k} \gamma_{k}=0 \\
& \left(1-h_{1}\right) \gamma_{k+1}+\ldots+\left(1-h_{l}\right) \gamma_{k+l}=
\end{aligned}
$$


For each $j=1,2, \ldots, l$, multiplication of the $j$-th equation by $h_{j}-1$ gives $\left(h_{j}-1\right)\left(h_{k+1}\right.$ $-1) \gamma_{k+j}=0$, i.e., $\left(h_{k+1}-1\right)\left(h_{j}-1\right) \gamma_{k+j}=0$, so that $\gamma_{k+j}=s_{j} \gamma_{k+j}^{\prime}$, for some $\gamma_{k+j}^{\prime} \in \mathbb{Z} G$, by (P2). Substitution of this value in the same equation yields $\gamma_{j}=\left(h_{j}-1\right) \gamma_{j}^{\prime}-\left(h_{k+1}-1\right) \gamma_{k+j}^{\prime}$, for some $\gamma_{j}^{\prime} \in \mathbb{Z} G$, by (P1). Also, for each $j^{\prime}=l+1, l+2, \ldots, k, \gamma_{j^{\prime}}=\left(h_{j^{\prime}}-1\right) \gamma_{j^{\prime}}^{\prime}$, for some $\gamma_{j^{\prime}}^{\prime} \in \mathbb{Z} G$. Hence $\gamma=\sigma\left(\sum_{s=1}^{k+l} \beta_{s} \gamma_{s}^{\prime}\right)$, so that (i) holds.

The verifications of (ii) and (iii) are similar and easier, and so are left out.

\section{Integral Homology and Cohomology of G.}

$$
\begin{aligned}
& H_{0}(G, \mathbb{Z}) \cong \mathbb{Z}, \\
& H_{1}(G, \mathbb{Z}) \cong \mathbb{Z} \oplus \mathbb{Z}_{m_{1}} \oplus \ldots \oplus \mathbb{Z} m_{k}, \\
& H_{2 r}(G, \mathbb{Z}) \cong \mathbb{Z}_{m_{1}} \oplus \ldots \oplus \mathbb{Z} m_{l}, \\
& H_{2 r+1}(G, \mathbb{Z}) \cong \mathbb{Z}_{m_{1}} \oplus \ldots \oplus \mathbb{Z} m_{k},
\end{aligned}
$$

for each $r \geqq 1$.

$$
\begin{aligned}
& H^{0}(G, \mathbb{Z}) \cong \mathbb{Z}, \\
& H^{1}(G, \mathbb{Z}) \cong \mathbb{Z}, \\
& H^{2 r}(G, \mathbb{Z}) \cong \mathbb{Z} m_{1} \oplus \ldots \oplus \mathbb{Z} m_{k}, \\
& H^{2 r+1}(G, \mathbb{Z})=\mathbb{Z} m_{1} \oplus \ldots \oplus \mathbb{Z} m_{l},
\end{aligned}
$$

for each $r \geqq 1$.

3.

We next consider a group $G$ given by generators: $h_{1}, h_{2}$; relations: $h_{1}^{m_{1}}=e, h_{1} h_{2}^{m_{2}}$ $=h_{2}^{m_{2}} h_{1}$.

If $m_{1}=m_{2}=2$, then $G \cong c m([4]$, p. 136).

\section{Definition 2.}

$$
\cdots \stackrel{\sigma}{\rightarrow} Y \stackrel{\iota}{\rightarrow} Y \stackrel{\sigma}{\rightarrow} Y \stackrel{\tau}{\rightarrow} Y \stackrel{\sigma}{\rightarrow} Y \stackrel{d_{2}}{\rightarrow} Y \stackrel{d_{1}}{\rightarrow} Y \stackrel{d_{0}}{\rightarrow} \mathbb{Z} G \stackrel{\varepsilon}{\rightarrow} \mathbb{Z} \rightarrow 0
$$

is a sequence of right $\mathbb{Z} G$-modules and $\mathbb{Z} G$-homomorphisms defined as follows:

$Y$ is a right $\mathbb{Z} G$-module free on $\alpha_{1}, \alpha_{2}$; and the homomorphisms are given by

$$
\begin{aligned}
& \varepsilon(g)=1 \in \mathbb{Z}, \text { for each } g \in G, \text { and extended by linearity; } \\
& d_{0}\left(\alpha_{i}\right)=h_{i}-1, \quad i=1,2
\end{aligned}
$$




$$
\begin{aligned}
& d_{1}\left(\alpha_{1}\right)=\alpha_{1} s_{1}, d_{1}\left(\alpha_{2}\right)=\alpha_{1}\left(h_{2}^{m_{2}}-1\right)+\alpha_{2} s_{2}\left(1-h_{1}\right) ; \\
& d_{2}\left(\alpha_{1}\right)=\alpha_{1}\left(h_{1}-1\right), d_{2}\left(\alpha_{2}\right)=-\alpha_{1}\left(h_{2}^{m_{2}}-1\right)+\alpha_{2} s_{1} ; \\
& \sigma\left(\alpha_{1}\right)=\alpha_{1} s_{1}, \sigma\left(\alpha_{2}\right)=\alpha_{1}\left(h_{2}^{m_{2}}-1\right)+\alpha_{2}\left(h_{1}-1\right) ; \\
& \tau\left(\alpha_{1}\right)=\alpha_{1}\left(h_{1}-1\right), \tau\left(\alpha_{2}\right)=-\alpha_{1}\left(h_{2}^{m_{2}}-1\right)+\alpha_{2} s_{2} ;
\end{aligned}
$$

and each extended by $\mathbb{Z} G$-linearity.

Theorem 2. The sequence (4) is a free $\mathbb{Z} G$-resolution of $\mathbb{Z}$.

Proof. It is easy to see that (4) extends (1).

As in Theorem 1 , we shall only verify that $\operatorname{Ker} d_{1} \subseteq \operatorname{Im} d_{2}$.

Let $\gamma=\alpha_{1} \gamma_{1}+\alpha_{2} \gamma_{2} \in \operatorname{Ker} d_{1}$. Then,

$$
\begin{aligned}
& s_{1} \gamma_{1}+\left(h_{2}^{m_{2}}-1\right) \gamma_{2}=0 \\
& s_{2}\left(1-h_{1}\right) \gamma_{2}=0 .
\end{aligned}
$$

Multiplication of the second equation by $h_{2}-1$ and subsequent application of P2 gives

$$
\gamma_{2}=s_{1} \gamma_{2}^{\prime}
$$

for some $\gamma_{2}^{\prime} \in \mathbb{Z} G$.

Substitution of this value in the first equation yields

$$
\gamma_{1}=\left(h_{1}-1\right) \gamma_{1}^{\prime}-\left(h_{2}^{m_{2}}-1\right) \gamma_{2}^{\prime}
$$

for some $\gamma_{1}^{\prime} \in \mathbb{Z} G$, by $\mathbf{P} 1$.

Hence $\gamma=d_{2}\left(\alpha_{1} \gamma_{1}^{\prime}+\alpha_{2} \gamma_{2}^{\prime}\right)$. Thus, $\operatorname{Ker} d_{1} \subseteq \operatorname{Im} d_{2}$.

Integral Homology and Cohomology of $G$.

$$
\begin{aligned}
& H_{0}(G, \mathbb{Z}) \cong \mathbb{Z}, \\
& H_{1}(G, \mathbb{Z}) \cong \mathbb{Z} \oplus \mathbb{Z} m_{1}, \\
& H_{l}(G, \mathbb{Z}) \cong \mathbb{Z} m_{1}, \text { for each } l \geqq 2 . \\
& H^{0}(G, \mathbb{Z}) \cong \mathbb{Z}, \\
& H^{1}(G, \mathbb{Z}) \cong \mathbb{Z}, \\
& H^{l}(G, \mathbb{Z}) \cong \mathbb{Z} m_{1}, \text { for each } l \geqq 2 .
\end{aligned}
$$


4.

We finally consider a group $G$ given by generators: $h_{1}, h_{2}$; relations: $\left(h_{1} h_{2}\right)^{2}=\left(h_{1}^{-1} h_{2}\right)^{2}$ $=e$. Then $G \cong p g g([4]$, p. 136).

\section{Definition 3.}

$$
\cdots \stackrel{\sigma}{\rightarrow} Y \stackrel{\leftarrow}{\rightarrow} Y \stackrel{\sigma}{\rightarrow} Y \stackrel{\tau}{\rightarrow} Y \stackrel{\sigma}{\rightarrow} Y \stackrel{d_{1}}{\rightarrow} Y \stackrel{d_{0}}{\rightarrow} \mathbb{Z} G \stackrel{\varepsilon}{\rightarrow} \mathbb{Z} \rightarrow 0,
$$

is a sequence of right $\mathbb{Z} G$-modules and $\mathbb{Z} G$-homomorphisms defined as follows:

$Y$ is a right $\mathbb{Z} G$-module free on $\alpha_{1}, \alpha_{2}$, and the homomorphisms are given by

$$
\begin{aligned}
& \varepsilon(g)=1 \in \mathbb{Z}, \text { for each } g \in G, \text { and extended by linearity; } \\
& d_{0}\left(\alpha_{i}\right)=h_{i}-1, \quad i=1,2 ; \\
& d_{1}\left(\alpha_{1}\right)=\alpha_{1} h_{2}\left(h_{1} h_{2}+1\right)+\alpha_{2}\left(h_{1} h_{2}+1\right), d_{1}\left(\alpha_{2}\right)=-\alpha_{1}\left(h_{1}^{-1} h_{2}+1\right)+\alpha_{2}\left(h_{1}^{-1} h_{2}+1\right) ; \\
& \sigma\left(\alpha_{1}\right)=\alpha_{1}\left(h_{1} h_{2}-1\right), \sigma\left(\alpha_{2}\right)=\alpha_{2}\left(h_{1}^{-1} h_{2}-1\right) ; \\
& \tau\left(\alpha_{1}\right)=\alpha_{1}\left(h_{1} h_{2}+1\right), \tau\left(\alpha_{2}\right)=\alpha_{2}\left(h_{1}^{-1} h_{2}+1\right) ;
\end{aligned}
$$

and each extended by $\mathbb{Z} G$-linearity.

Theorem 3. The sequence (6) is a free $\mathbb{Z} G$-resolution of $\mathbb{Z}$.

Proof. As before we shall only verify that $\operatorname{Ker} d_{1} \subseteq \operatorname{Im} \sigma$. Let $\gamma=\alpha_{1} \gamma_{1}+\alpha_{2} \gamma_{2} \in \operatorname{Ker} d_{1}$. Then

$$
\begin{gathered}
h_{2}\left(h_{1} h_{2}+1\right) \gamma_{1}-\left(h_{1}^{-1} h_{2}+1\right) \gamma_{2}=0, \\
\left(h_{1} h_{2}+1\right) \gamma_{1}+\left(h_{1}^{-1} h_{2}+1\right) \gamma_{2}=0 .
\end{gathered}
$$

Adding the equations, we have $\left(h_{2}+1\right)\left(h_{1} h_{2}+1\right) \gamma_{1}=0$, so that $\gamma_{1}=\left(h_{1} h_{2}-1\right) \gamma_{1}^{\prime}$, for some $\gamma_{1}^{\prime} \in \mathbb{Z} G$, by (P1) and the fact that $h_{2}$ has infinite order. Substitution in the second equation gives $\left(h_{1}^{-1} h_{2}+1\right) \gamma_{2}=0$, and so, $\gamma_{2}=\left(h_{1}^{-1} h_{2}-1\right) \gamma_{2}^{\prime}$, for some $\gamma_{2}^{\prime} \in \mathbb{Z} G$. Thus, $\gamma$ $=\sigma\left(\alpha_{1} \gamma_{1}^{\prime}+\alpha_{2} \gamma_{2}^{\prime}\right)$, and so $\operatorname{Ker} d_{1} \subseteq \operatorname{Im} \sigma$.

\section{Integral Homology and Cohomology of G.}

$$
\begin{aligned}
& H_{0}(G, \mathbb{Z}) \cong \mathbb{Z}, \\
& H_{1}(G, \mathbb{Z}) \cong \mathbb{Z}_{2} \oplus \mathbb{Z}_{4}, \\
& H_{2 l}(G, \mathbb{Z}) \cong 0, \\
& H_{2 l+1}(G, \mathbb{Z}) \cong \mathbb{Z}_{2} \oplus \mathbb{Z}_{2},
\end{aligned}
$$

for each $l \geqq 1$. 


$$
\begin{aligned}
& H^{0}(G, \mathbb{Z}) \cong \mathbb{Z}, \\
& H^{1}(G, \mathbb{Z}) \cong 0, \\
& H^{2}(G, \mathbb{Z}) \cong \mathbb{Z}_{2} \oplus \mathbb{Z}_{4}, \\
& H^{2 l-1}(G, \mathbb{Z}) \cong 0, \\
& H^{2 l}(G, \mathbb{Z}) \cong \mathbb{Z}_{2} \oplus \mathbb{Z}_{2},
\end{aligned}
$$

for each $l \geqq 2$.

Acknowledgment. I express my sincere thanks to the referee for his valuable suggestions.

\section{REFERENCES}

1. S. Majumdar, A free resolution for a class of groups, J. Lond. Math. Soc. (2) 2 (1970).

2. A. M. Macbeath, Discontinuous groups and bilateral transformations, Proceedings of the Summer School in Geometry and Topology (Queen's College, University of St. Andrews, 1961).

3. A. Best, On the Existence of Fuchsian Groups with Compact Orbit Spaces (M.Sc. Thesis, University of Birmingham, 1964).

4. H. S. M. COXeter and W. O. Moser, Generators and Relations for Discrete Groups (Springer-Verlag, Berlin, 1957).

5. R. C. Lyndon, Cohomology theory of groups with a single defining relation, Ann. of Math. $52(1950)$.

6. A. Learner, Cohomology of Groups (Lecture Notes, Queen Mary College, University of London, 1965).

7. R. B. Fox, Free differential calculus I, Ann. of Math. 57 (1953).

RAJSHAHI UNIVERSITY

BANGLADESH

Present address:

MaKeRE UNIVERSITY

P.O. Box 7062

Kampala, UGanda 